the shock type (E.C.T. or insulin) of therapy does produce results whether Dr. Winnicott wishes to believe it or not.

My personal complaint is that this schism tends to split psychiatry into two camps. Surely the proper training should consist in a basis of psychotherapy for the neuroses and a knowledge of electrical and insulin treatment for those who need it. Unfortunately there are many who, in their ignorance, have come to despise psychotherapy and delight in their shining machines and glittering syringes. In the same way the more conservative of the older psychiatrists refuse to countenance the occasion for good in the new ways and curse the young for whoring after strange gods. It seems to me that the simple secret of cure is the ability to diagnose the psychiatric condition and the broad-mindedness to use the right method, whether shock or psychological. Such success as has come my way has come from this.

Now may I offer a criticism of those who use shock treatments ? First, they do not cure such illnesses as homosexuality, fetishisms, hysteria, and simple neuroses, yet I have seen them uselessly applied. Secondly, only this week a woman came to me who feared to have E.C.T. because she had been hustled into a side room all ready for the machine as soon as the preceding patient had been whipped off. Unfortunately she heard the epileptic cry, and this unearthly sound haunted her so that she feared to have further shocks. I have seen similar patients. It is a piece of unnecessary cruelty to allow such a thing to happen whether we believe that mental illness is the result of a "subacutely or chronically diseased physical organ called cerebrum " or not. Lastly, may I suggest that leucotomy should be reserved as a forlorn hope for those who have failed to respond to E.C.T., insulin, and perhaps prolonged narcosis? It has a significant death rate, and severe injury to the personality may result even if no intellectual damage does result.

Lest it be thought that I am hostile to organic forms of treatment may I record a case which passed through my hands many years ago and which suggests that there may be undreamed of forms of treatment? This was a middle-aged woman with mitral disease. She was so grossly hallucinated that she kept the ward echoing with her shouts of abuse. Her days were hell and her nights a torment. Then one day she developed an infarct in the left side of her brain, with a resulting right hemiplegia and motor aphasia. The "voices" stopped instantly as if disconnected. When she was recovered enough to be able to express herself she said that it had been truly magical. Perhaps one day a surgeon will be able to repeat such magic at will-but we shall still need psychotherapists for the neurotics.-I am, etc.,

London, W.1.

Clifford Allen.

SIR,-Psychiatrists in general and all those interested in the empirical aspect of psychiatric research, particularly on the therapeutic side, will view with some misgiving the prominence afforded to the criticisms of the physical treatments of mental disease outlined by Dr. D. W. Winnicott (May 17, p. 688). If my memory is correct, Dr. Winnicott has already voiced similar criticisms in the correspondence section of the British Medical Journal, where viewpoints do not lose their purely personal touch. When, however, these personal opinions are promoted to the importance of a special article, they tend to assume the imprimatur of authority and are consequently liable to be accepted as unimpeachable expert opinion based on extended and specialized experience. They must, on this interpretation, create widespread misconception among non-psychiatric medical colleagues, particularly when they are of an exclusively destructive nature as in this case.

Dr. Winnicott's opinions, apparently given originally to the medical section of the British Psychological Society, would appear to constitute, in a rather defensive fashion, the viewpoint and attitude of the purely psychological school of thought towards mental disease, as represented actively by the psychoanalysts. To the latter any encroachment, with a tendency to monopoly, by the physical therapists must appear in the light of rank heresy and be denounced as " empirical magic." Every experienced psychiatrist admits the necessity of recognizing both psychological and physical factors in mental disease and wisely combines both in his therapeutic approach. The American authority, Dr. A. P. Noyes, emphasizes this point in his Modern Clinical Psychiatry as follows:
"No one formulation, whether it be chemical, physiological, neurological or psychological, can explain all the phenomena observed in those personality disorders that we call mental disease We must remember that much of our talk about the unconscious, about organic lesions and about disturbed chemistry is as yet hypothetical. In psychiatry we deal with the socialiy conditioned biopsychic life of the individual, and the biopsychic life, it is obvious, is subject to an incalculable variety of influences."

E.C.T., which has been mainly attacked by Dr. Winnicott and, rather flippantly, on the grounds that psychiatrists do not use it on themselves, may not, in many cases, effect a cure per se, and is generally followed up with a combination of psychotherapy and occupational therapy, as here in Killarney Mental Hospital. All three create a reinforcing effect in the therapeutic adjustment of both the psychic and physical elements of the disorder under treatment. In actual fact, E.C.T. effects a very high percentage of recoveries in the true uncomplicated depressive psychoses. particularly the involutional types. The high percentage rate of recovery is such that the criticism of Dr. Winnicott, even though admittedly based on "personal prejudice," will not cause any undue anxiety among practising psychiatrists. Further, the efficacy of E.C.T. in the case of all confusional psychoses of toxic origin, whether puerperal or otherwise, is of such a nature as to be regarded as specific.

Dr. Winnicott's reference to " organized paranoia" would suggest, on his part, some confusion in nomenclature as well as practice. Quite obviously, from his ensuing remarks, he is confusing true paranoia with the paranoid form of schizophrenia, and his suggestion of the indiscriminate use of E.C.T. for this latter disease is entirely contrary to recent practice. It is now generaliy admitted that unmodified E.C.T. for any form of schizophrenia does not effect a cure even in the acute cases. This is our experience in this hospital, though there have been very encouraging results in the administration of E.C.T. following thyroxine premedication in acute and recent cases. In one such case, where a full course of deep insulin therapy here had been a failure, there was a very successful result in the modified form of E.C.T. described.

All treatments, whether physical or psychological, will be iudged on the following considerations: (a) good results achieved, $(b)$ the permanency of these results, and $(c)$ absence of injurious effects, whether immediate or residual. (a) The results in the conditions already outlined cannot be questioned on any extended experience. (b) Present experience goes to show that there is a very high degree of permanency in the results achieved for the conditions mentioned above. Even in the very small number of reapsing cases, hospitalization is reduced to a very considerable extent as against control cases. (c) There are no immediate ill-effects that would contraindicate E.C.T., and the alleged brain damage has not been borne out in the vast number of cases that have been submitted for the treatment. It is on these three grounds that.E.C.T. has been accepted as a very definite therapeutic advance in psychiatry, even Dr. Winnicott admits rather pointlessly, "I have no hope that these arguments will make any sudden difference to the now established practice of psychiatry."

There are many other points in Dr. Winnicott's article, as absurd as they are flippant, that do not merit recognition, much less contradiction. There is, however, reference to mental hospital management in the penultimate paragraph of his article which is entirely contrary to fact. Probably in no form of nursing are the qualities of forbearance, patience, and understanding so necessary and still so generally exercised as in psychiatry, and to suggest that it is permeated with hatred and cruelty casts a very objectionable reflection on modern mental nursing. This unfounded charge is no less absurd than the "sympathetic" consideration expressed for the doctors and nurses whose protection from developing "emotional instability" in the cause of their work may be seriously threatened by the " abolition of shock therapy." Does there exist a rational being who is convinced of either the validity or the sanity of such arrant nonsense? Dr. Winnicott apparently thinks there does.-I am, etc.,

Killarney, Eire.

Eamon N. M. O'Sullivan.

\section{Tuberculosis and Diabetes Mellitus}

SIR,-I should like to associate myself with Dr. Wilfrid Oakley's plea (May 31, p. 780) for sanatoria where efficient management of diabetes could be given to diabetics being treated for pulmonary tuberculosis. The tuberculous diabetic does not often receive adequate treatment for both his diseases, and it is therefore not surprising that many of these patients fare badly. It is essential that both diseases receive proper consideration, and until special centres are created to ensure the 
correct management of both maladies the results will not give hope and encouragement to sufferers or their medical advisers.

The tuberculosis service in this country may have reached as high a peak of perfection as it has ever attained since its inauguration, but this will hardly avail the diabetic unless his metabolic disturbance be at the same time under expert supervision. Experience has shown that good treatment of the pulmonary disease with inadequate supervision of the diabetes is as harmful as poor treatment of the phthisis with proper control of the diabetes. It goes without saying that bad treatment of both conditions is inevitably disastrous. The truth is that in many instances the tuberculous diabetic is uncared for according to the best medical standards, and the evil effects of this are brought to our notice in the published results of treatment.

At the few clinics where adequate control of both diseases has been enforced from the beginning the results have indicated that the outlook for the diabetic who develops phthisis hardly differs from that of the non-diabetic. Figures published from sanatoria in the past have led to false conclusions as to the seriousness of the combination of these two diseases; the bad results obtained were probably due to inadequate control of both conditions according to modern therapeutic standards. Unfortunately this fact has not been fully realized, with the result that the tuberculous diabetic has acquired a stigma of hopelessness based on mismanagement of his diseases.

Publication of the new version of Section I of the Appendix to Memorandum 37/T (Revised), which has been accepted by the Ministry of Health (Supplement, May 31, p. 111), raises important considerations concerning the tuberculous diabetic. In the section on the classification of patients suffering from tuberculosis, it is stated that "all cases with grave complications, whether they are tuberculous or not, should be classified in this group [group B3] (e.g., diabetes, tuberculosis of intestine or larynx)." My first objection to this new classification is that it places the diabetic whose disease is complicated by a small pulmonary lesion in the same category as the patient with advanced bilateral fibro-cavernous disease. Secondly, the presence of diabetes is looked upon in the same light as tuberculous enteritis or laryngitis. Here, surely, a gross injustice is done to the diabetic. Whereas the onset of tuberculous enteritis in most cases heralds a fatal issue, the outlook for the tuberculous diabetic, as emphasized already, is never as serious as this unless by virtue of the extent of his pulmonary disease. The combination of diabetes and tuberculosis should never be regarded as the equivalent of hopeless and irrecoverable disease.

Apart from the objections on medical grounds to placing tuberculous diabetics in the class B3 one can imagine many othęr disadvantages that such a patient may suffer owing to his consideration in this group. B3 cases have always been regarded as suffering from advanced pulmonary disease, so that there has been no urgency in considering their admission to sanatoria for treatment. They may be left at home, if conditions allow, or they may be admitted to homes or hospitals for advanced cases. It would be most unfortunate if tuberculosis officers should be led, after reading the new classification, to treat their tuberculous diabetics in a like manner. The development of phthisis as a complication of diabetes should be looked upon as a medical emergency, requiring prompt treatment for both conditions. An unnecessarily long wait for admission can only mean advance of the pulmonary disease together with inadequate control of the diabetes and a poor outlook for the patient; accordingly, such patients warrant priority on the waiting-lists. Finally, are the benefits of the maintenance grants from the Treasury (Memo 266/T) to be withheld from tuberculous diabetics under the new scheme of classification?

The diabetic surely has to suffer enough already on account of his unfortunate affliction without having additional difficulties put in his way which are based merely on prejudice and receive no support from properly instituted clinical studies. It is to be most earnestly hoped that further consideration may be given to the amended Section I, wherein diabetics are included in class B3, and that special units will soon be established for the treatment of tuberculous diabetics.-I am, etc.,

\section{Classification of Tuberculous Patients}

SIR,-Dr. H. E. Stevens's letter (May 31, p. 782) draws attention to a deficiency in the new classification of tuberculosis terms recently adopted by the Ministry of Health. The object of adopting the new classification is not stated, but presumably it is mainly to secure increased accuracy of returns through clarification of the headings used in compiling them. My own belief is that this object will not be attained by the present proposals, and if other tuberculosis workers feel the same they might consider whether it is worth their while to ask for a re-examination of the scheme now put forward.

The following are some of the grounds on which I base my opinion :

(1) The replacement of the self-explanatory terms "minus" and "plus" by A and B is to me a retrograde step, and is bound to lead to mistakes in classification owing to the absence of any apparent relation of the letters $\mathrm{A}$ and $\mathrm{B}$ with either positive or negative cases.

(2) The definitions of respiratory and non-respiratory tuberculosis are not free from criticism. One expects these definitions to be helpful to the extent that few, if any, occasions will arise when it will be impossible to classify a patient by their use. But such occasions are bound to happen when no provision is made in the definitions for including conditions other than those specified-for example, endo-bronchial tuberculosis. The difficulty would be overcome by substituting the words "a respiratory case includes ..." for the words " a respiratory case should be. ..."

(3) A "combined" case-e.g., with pulmonary and glandular tuberculosis-is classified as a pulmonary case, as at present. But if such a patient is found to have tubercle bacilli in his excised glands he will be classified as $B$, giving the impression that he is infectious in the same degree as a patient who is discharging tubercle bacilli in his sputum. The definition of an infectious pulmonary case is incomplete, like the general definition of a pulmonary case. The finding of tubercle bacilli should be more definitely related to the pulmonary lesion by specifying the situations where the bacilli will be regarded as evidence of their pulmonary origin.

(4) The definition of a class B case suffers from the further defect that it makes no provision for reclassifying a recovered case who comes on the dispensary register again after a period of years. According to the literal application of the wording, a person who had tuberculous cervical glands in childhood with tubercle bacilli in the pus from the glands, and who comes on the register with any tuberculous condition 20 years later, is to be called a $B$ case. For the purposes of compiling uniform statistics this procedure may be very desirable, but the statistics themselves can hardly be helpful. I submit that a definition of a "return" or "restored" case is required, and that the classification of such a case should be based on the current findings and not on the bacteriological results obtained many years previously.

(5) The subsections of paragraph III of the memorandum are inconsistent. Subsection (2) states that respiratory A.s and B.s should be subdivided; subsection (3) states that they should be further subdivided. Yet there is only one basis of subdivision described-viz., into groups 1,2 , and 3 . The meaning is clear enough, but the wording is confusing.

(6) The definition of "quiescent" does not make any allowance for variations in bacteriological control by different observers: some will rely on repeated slide examinations, some will prefer culture or inoculation methods. But, according to the definition, unless three consecutive monthly slides are negative, the case does not fulfil the criteria required for quiescence. Moreover the definition does not take account of the fact that a patient may not be able to produce sputum when required at monthly intervals, and it seems clear that it cannot be strictly observed as it stands at present.

(7) The classification "arrested" does not seem to serve any useful purpose. In practice it is never used to describe any patient, and rather than set down details for its interpretation I consider it should be abolished.

(8) Does the definition of a "recovered" case apply only to pulmonary patients? If it is intended to apply to nonpulmonary patients also, then it should be stated, as at present a non-pulmonary case can be written off as "recovered" after three years.

(9) Regarding the definition of "active case," I consider it unfortunate that, by implication, all patients not positive within the preceding three months may be taken to be classifiable as not active-i.e., quiescent. I am sure this is not the intention, but the wording is not so helpful as it might be.

(10) The use of the word "stationary" to denote a case in which the patient remains in statu quo appears unscientific and unnecessary, for if a patient was "quiescent" at the beginning 